\title{
DIVERSIDADE DE COLEOPTERA (INSECTA) PRESENTE NO CENTRO DE FORMAÇÃO LARANJAL NO MUNICÍPIO DE ABAETETUBA-PA
}

\author{
DIVERSITY OF COLEOPTERA (INSECTA) PRESENT AT THE ORANGE TRAINING CENTER IN THE \\ MUNICIPALITY OF ABAETETUBA-PA
}

\section{DIVERSIDAD DE COLEOPTERA (INSECTA) PRESENTE EN EL CENTRO DE FORMACIÓN NARANJA DEL MUNICIPIO DE ABAETETUBA-PA}

\author{
Edison Cardoso Pinheiro Júnior ${ }^{1}$ (D) \\ Natanael Charles da Silva ${ }^{2 *}$ (D)
}

\begin{abstract}
${ }^{1}$ Graduado em Licenciatura em Ciências Biológicas, Instituto Federal do Pará (IFPA), Abaetetuba, Pará, Brasil. ${ }^{2}$ Doutorando em Ensino de Ciências e Matemática, Universidade Federal do Rio Grande do Norte (UFRN), docente pelo Instituto Federal do Pará (IFPA), Abaetetuba, Pará, Brasil; *Autor correspondente: natanaelcharles@gmail.com.
\end{abstract}

Recebido: 03/08/2021 | Aprovado: 20/08/2021| Publicado: 24/08/2021

\begin{abstract}
Resumo: Compondo a ordem de insetos com maior número de representantes e diversidade, os coleópteros possuem grande importância ecológica e social. Baseado nisso, o objetivo deste estudo foi conhecer a diversidade de coleópteros presentes em um fragmento florestal no município de Abaetetuba-PA considerando dois métodos de coleta diferentes. As coletas ocorreram em um intervalo de seis meses utilizando armadilhas do tipo pitfall como método de coleta passiva e o puçá entomológico como método de coleta ativa. Depois de coletados, os espécimes foram fixados em uma câmera mortífera, identificados e armazenados. As armadilhas pitfall capturaram um quantitativo maior de espécimes, mesmo sendo dividida em borda e centro. As famílias Erotylidae, Cerambycidae e Tenebrionidae foram as que se mostraram menos representativas e as famílias Scarabaeidae e Chrysomelidae foram as mais representativas nos dois ambientes. Com o estudo, verifica-se que embora haja diferença no quantitativo de espécimes coletados nos diferentes ambientes (borda, centro e coleta ativa), os índices de diversidade entre estes locais não expressam valores com diferenças significativas.
\end{abstract}

Palavras-chave: Insetos. Fragmentação florestal. Besouros.

Abstract: Composing the order of insects with the greatest number of representatives and diversity, beetles have great ecological and social importance. Based on that, the objective of this study was to know the diversity of beetles present in a forest fragment in the city of Abaetetuba-PA, considering two different collection methods. The collections took place at an interval of six months using pitfall traps as a passive collection method and the entomological puça as an active collection method. After being collected, the specimens were fixed in a deadly camera, identified and stored. The pitfall traps captured a larger quantity of specimens, even being divided into edge and center. The Erotylidae, Cerambycidae and Tenebrionidae families were the least representative and the Scarabaeidae and Chrysomelidae families were the most representative in both environments. With the study, it appears that although there is a difference in the quantity of specimens collected in different environments (border, center and active collection), the diversity indices between these places do not express values with significant differences.

Keywords: Insects. Forest fragmentation. Beetles.

Resumen: $\mathrm{Al}$ componer el orden de insectos con mayor número de representantes y diversidad, los escarabajos tienen una gran importancia ecológica y social. En base a eso, el objetivo de este estudio fue conocer la diversidad de escarabajos presentes en un fragmento de bosque en la ciudad de Abaetetuba-PA, considerando dos métodos de recolección diferentes. Las recolecciones se llevaron a cabo en un intervalo de seis meses utilizando trampas de caída como método de recolección pasiva y la puça entomológica como método de recolección activa. Después de ser recolectados, los especímenes fueron fijados en una cámara mortal, identificados y almacenados. Las trampas de caída capturaron una mayor cantidad de ejemplares, incluso dividiéndose en borde y centro. Las familias Erotylidae, Cerambycidae y Tenebrionidae fueron las menos representativas y las familias Scarabaeidae y Chrysomelidae fueron las más representativas en ambos ambientes. Con el estudio, parece que si bien existe una diferencia en la cantidad de ejemplares recolectados en diferentes ambientes (borde, centro y colección activa), los índices de diversidad entre estos lugares no expresan valores con diferencias significativas. 
Palabras-clave: Insectos. Fragmentación forestal. Escarabajos.

\section{INTRODUÇÃO}

O termo coleóptero vem do termo grego koleos $=$ estojo e pteron $=$ asa, devido as asas anteriores endurecidas, sendo a transformação das asas anteriores em élitros, o fator mais determinante para o sucesso desta ordem (Rafael et al., 2012). Pois, geralmente os besouros possuem quatro asas, as mesotorácicas (anteriores) engrossadas e endurecidas (élitros), se encontrando divididas em uma linha reta ao longo da porção mediana do dorso, cobrindo as asas metatorácicas (posteriores) frágeis e membranosas, que em repouso permanecem dobradas embaixo do élitro (Casari \& Ide, 2012).

Apresentando notável relevância em áreas verdes, os insetos são um grupo taxonômico muito estudado em razão de sua sensibilidade às alterações climáticas, sendo considerados bioindicadores ambientais (Spiller, Spiller \& Garlet, 2018). Por esta característica, eles recebem maior destaque, considerando ainda, suas variedades de hábitos alimentares, nichos ecológicos, riqueza de famílias e diversidade de espécies (Teixeira, Hoffmann \& Silva Filho, 2009).

Com relação a diversidade presente em um fragmento florestal, a desordem neste ambiente provocado por diversos fatores pode levar à perda de habitats, além da degradação de solos que restringe a recuperação de florestas secundárias, as quais podem se tornar menos diversas (Pedro Júnior, Murrieta \& Adams, 2008). Sobre este fato, França \& Marini (2009) afirmam que a fragmentação de florestas constitui uma das maiores ameaças à perda da biodiversidade, gerando problemas graves relacionados a variação de espécies de florestas tropicais como a amazônica. Desta forma, as atividades antrópicas degradam os ecossistemas e desestruturam as bases ecológicas do planeta (Couto, 2020).

Nos últimos anos, os principais estudos que visam o entendimento sobre a diversidade em escala de paisagem, buscam compreender como a quantidade de habitat (composição) e a fragmentação (configuração) alteram diferentes aspectos da diversidade (Rodríguez et al., 2016; Santos et al., 2017). Baseando-se nisso, o objetivo deste estudo foi conhecer a diversidade de coleópteros presentes em um fragmento florestal situado no município de Abaetetuba-PA considerando dois métodos de coletas.

\section{1 importância e diversidade dos Coleópteros (Insetos)}

Nas regiões tropicais e subtropicais, a pressão da caça e a destruição dos hábitats naturais, causados principalmente por pressões do crescimento populacional e atividades econômicas são os principais responsáveis pela perda da biodiversidade (López et al. 2017). Em função da riqueza de ecossistemas, Martins \& Oliveira (2015) argumentam que o Brasil é considerado o país com maior diversidade de vida existente, contando com sete biomas, dentre eles a Amazônia, que apresenta forma peculiar de fauna e flora contribuindo para a riqueza de espécies do país. 
No entanto, estudos científicos como o de Souza Junior et al. (2013) vêm demonstrando a degradação das florestas na Amazônia, assim como os impactos sobre a biodiversidade e os serviços ecológicos (Berenguer et al., 2014; Moura et al., 2013). As atividades antrópicas, por exemplo, decorrentes do crescimento econômico, como a construção de estradas, atividades industriais e agrícolas mal planejadas e a formação de pastagens, tem resultado em um considerável aumento na degradação ambiental no país (Machado et al., 2013; Hora, Fonseca \& Sodré, 2015). Além destas, o desmatamento pode ser caracterizado como um dos principais problemas relacionado ao meio ambiente (Brasil, 2016).

Com isso, a substituição de florestas contínuas por fragmentos pequenos e isolados diminui a capacidade de sobrevivência das populações, principalmente porque o isolamento dificulta as taxas de recolonização (Metzger, 2012). Portanto, essa fragmentação florestal ocasiona redução da diversidade de fauna e aumento na taxa de mortalidade de árvores (Laurance \& Vasconcelos, 2009). Além disso, os estudos de Burivalova et al. (2015) e Moura et al. (2013) mostram que certas espécies da fauna e da flora são encontradas somente em florestas bem preservadas, desaparecendo após eventos de perturbação.

Nascimento et al. (2010) apontam que esses efeitos são mais significativos nas margens do fragmento, por estarem mais susceptíveis as alterações como a transição de uma mancha ou de um bioma por uma pastagem, espaço urbano, agricultura e outros fatores que interrompam abruptamente a paisagem. Lima (2012) demonstra em suas pesquisas que nas paisagens fragmentadas além da perda da biodiversidade, também ocorre diminuição dos biomas, aumento do efeito de borda, quebras dos fluxos ecológicos, mudanças na mortalidade e natalidade de espécies, alteração na polinização, especiação, competição, predação, mutualismo, perda da variedade genética e por fim, como consequência mais grave, a extinção das espécies.

Nesse contexto, os insetos podem ser considerados "espécies-chaves", pois a sua ausência e/ou perda de sua função ecológica poderia gerar um colapso no ecossistema (Gullan \& Cranston, 2017). Sua sensibilidade às mudanças e alterações ambientais afeta a riqueza, distribuição e abundância do grupo, acarretando em modificações nos padrões comportamental e na abundância sazonal destes indivíduos, que por sua vez, vêm sendo utilizados como ferramenta para explanar e tentar entender os distúrbios ambientais (Oliveira et al., 2014).

Considerando esta diversidade de funções e características dentro da classe insecta, podemos destacar as ordens Coleóptera (besouros), Lepidóptera (borboletas e mariposas), Orthoptera (grilos e gafanhotos) e Odonata (libélulas) por suas diversidades e suas qualidades bioindicadoras (Rafael et al., 2012). Destaca-se ainda, as ordens Hymenoptera (vespa, formigas, abelhas) e Isoptera (cupins), por formarem sociedades complexas e aos Diptera (moscas e pernilongos,) e Hemiptera (percevejos e barbeiros) por serem vetores de doenças (Gullan \& Cranston, 2017).

Os insetos bioindicadores, como os coleópteros, por exemplo, podem ser utilizados com a função específica de indicadores ambientais que respondam as perturbações ou mudanças no local, como indicadores ecológicos que demonstram efeitos das mudanças ambientais, como, alterações de habitats, fragmentação, mudanças climáticas, poluição ou até mesmo, outros fatores que geram impactos na biota ou ainda como 
indicadores da biodiversidade de fauna e flora do local (Silva \& Silva, 2011).

Como seus ciclos são geralmente curtos, eles podem multiplicar-se rapidamente em condições favoráveis (Silva \& Silva, 2011), apresentando desta forma, de acordo com Casari \& Ide (2012) papel importante na reciclagem de nutrientes, principalmente na fase larval, por isso, desempenham ainda grande êxito no controle biológico de insetos pragas e espécies de plantas nocivas.

Dentre as qualidades atribuídas à ordem Coleóptera para utilizá-la como bioindicadora ambiental, estão a grande diversidade de espécies e a grande abundância, associados ao fato de ocuparem os mais diversos nichos ecológicos (Gullan \& Cranston, 2017), além disso, eles podem se comportar como fitófagos, xilófagos, rizófagos, polinífagos, granívoros, micetófagos, coprófagos, necrófagos e carnívoros, sendo a hematofagia o único hábito alimentar não encontrado em indivíduos dessa ordem (Moreira, 2015).

Davis et al. (2001) salientam que em ecossistemas florestais, os coleópteros envolvidos no processo de ciclagem de nutrientes e dispersão de sementes, podem ser utilizados na avaliação dos efeitos de distúrbios florestais, pois, muitas famílias de coleópteros são altamente especializadas no nicho ecológico que ocupam (Wink et al., 2005). Segundo Marinoni (2001), as análises das interações ecológicas entre flora e fauna são estabelecidas com base nos hábitos alimentares, que na maioria dos coleópteros é semelhante em nível taxonômico de família ou subfamília.

Nesse sentido, Fernandes et al. (2011) consideram que estudos em níveis de família são importantes, pois podem ser usados para auxiliar a avaliação da qualidade ambiental, além de viabilizar, retratar e monitorar uma área com o uso da entomofauna bioindicadora (Carvalho, 2011). Desta forma, Carvalho (2011), Campos (2012) e Otavo (2013) afirmam que a família Scarabaeidae possui indivíduos que são afetados por intervenções humanas e que devido ao seu comportamento de natureza sedentária, se tornam vulneráveis às mudanças ambientais. Fernandes et al. (2011) apontam os estafilinídeos e silfídeos como bioindicadores, por apresentarem sensibilidade em relação à composição florística, nível de serapilheira e luminosidade. Já os lampirídeos, elaterídeos e fengodídeos, por possuírem bioluminescência e ao não serem observados em um estudo, sua ausência pode estar associada com o processo de urbanização, pois a iluminação artificial afeta sua ocorrência (Vivian, Rocha \& Hagen, 2010).

Além destas, Segundo Pimenta (2011) os crisomelídeos são potenciais bioindicadores por manterem uma estreita relação com suas plantas hospedeiras. Casari \& Ide (2012), Godinho Júnior (2011), Quinteiro, Lopes \& Martins (2012) e Triplehorn \& Johnnson (2011) indicam a importância de outras famílias de coleópteros bioindicadores, como: Coccinelidae cujo declínio pode ser caracterizado pela urbanização intensa e uso de agrotóxicos no local ou nas proximidades, Carabidae que podem indicar a presença de metais no solo, Curculionidae que se alimentam de tecidos vegetais, sendo considerados importantes pragas agrícolas e florestais e os Cerambicídeos que desempenham um papel importante na reciclagem da matéria vegetal morta (Rocha, 2010). 


\section{MATERIAL E MÉTODOS}

Para realização da pesquisa, solicitamos autorização ao Sistema de Autorização e Informação em Biodiversidade (SISBIO) e utilizamos como lócus de estudo uma área dentro do município de Abaetetuba, conhecida como Centro de Formação Laranjal (Figura 1), cuja sede foi fundada em 17 de abril de 1965 e encontra-se situada na Rodovia Dr. João Miranda PA-252, no bairro Cristo Redentor, perímetro urbano do município.

Figura 1 - A - Perímetro do município de Abaetetuba; B - Local de coleta (Centro de Formação Laranjal Abaetetuba-PA).

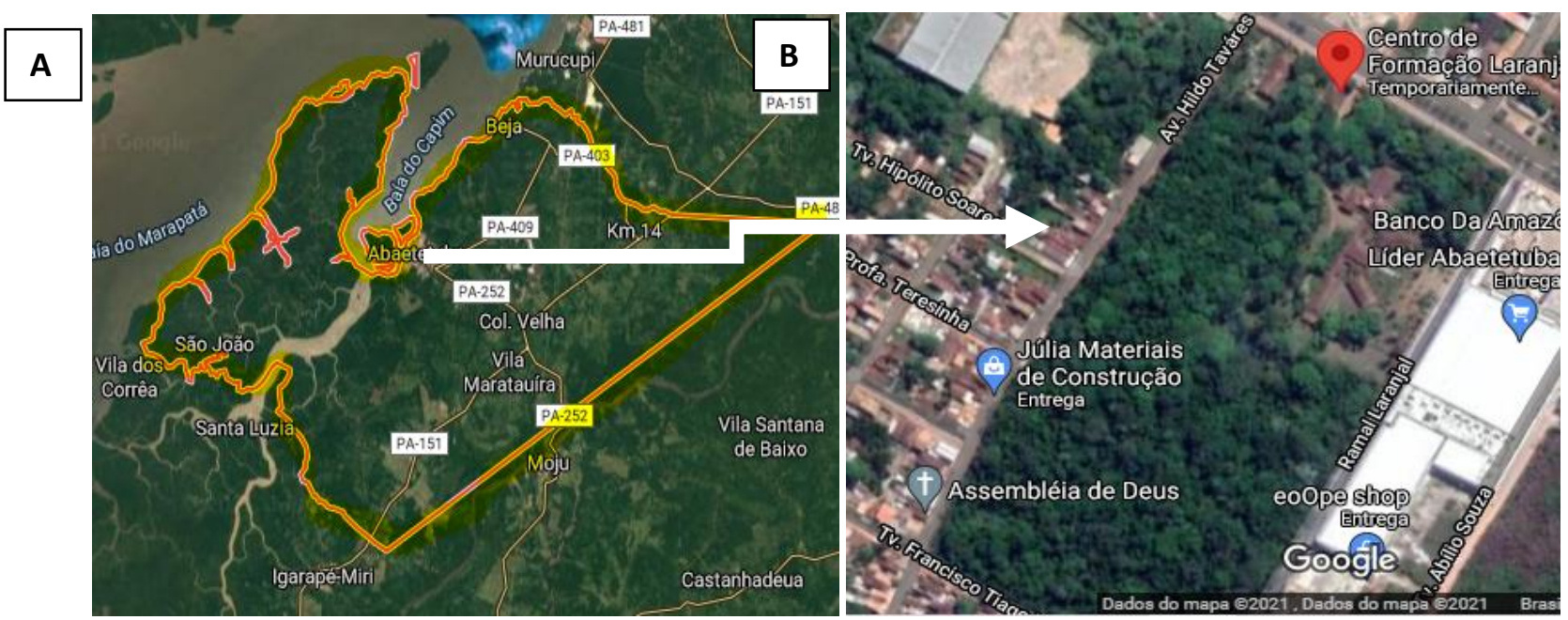

Fonte: Elaborado pelos autores (2021).

As coletas foram realizadas em um intervalo de seis meses (setembro de 2020 a fevereiro de 2021), que corresponde ao período de transição entre o verão e inverno amazônico, podendo com isso, identificar possíveis alterações entre a diversidade de coleópteros capturados nos dois períodos. Nas coletas foram utilizadas armadilhas do tipo pitfall (espalhadas tanto na borda do fragmento quanto no centro) e puçá entomológico (coletando em regiões também de borda e centro), as mesmas foram realizadas por um período de 3 horas, uma vez por semana de cada mês no período considerado, totalizando assim, um esforço amostral de 72 horas.

Após coleta, os espécimes foram fixados em uma câmera mortífera e levados ao Laboratório de Biodiversidade e Conservação (LABICON) para dar seguimento ao processo de identificação e armazenamento. A identificação deu-se até o nível de família, uma vez que estudos de diversidade utilizando famílias já foram realizados por Amado (2012), Garlet, Costa \& Boscardin (2016) e Saldanha (2020), utilizando para isso a chave dicotômica de Casari \& Ide (2012).

No cálculo da riqueza de famílias representado por "S", adaptamos a definição de Coelho (2000) para riqueza de espécies, calculando o Índice de Diversidade de Shannon-Wiener (H') e o índice de diversidade de Simpson ( $\lambda$ ) utilizando-se o programa estatístico Past, versão 2.17c (Hammer et al., 2013). Além disso, foi 
realizado o cálculo do teste t-Student entre as amostras coletadas no centro e borda com a finalidade de identificar diferenças na diversidade de coleópteros nestes ambientes.

\section{RESULTADOS E DISCUSSÃO}

Com o quantitativo de espécimes de coleópteros coletados por família (Figura 2) e por armadilhas do tipo pitfall em borda e centro e as coletadas por busca ativa, obtemos que as armadilhas pitfall capturaram um quantitativo maior de espécimes do que a coleta por busca ativa, mesmo sendo dividida em borda e centro, onde das famílias capturadas a Erotylidae, Cerambycidae e Tenebrionidae foram as que se mostraram menos representativas nesse estudo e não tiveram representantes capturados nas armadilhas pitfall.

Figura 2 - Representação dos espécimes por família coletadas com armadilhas do tipo pitfall de borda e centro e coleta ativa com puçá.

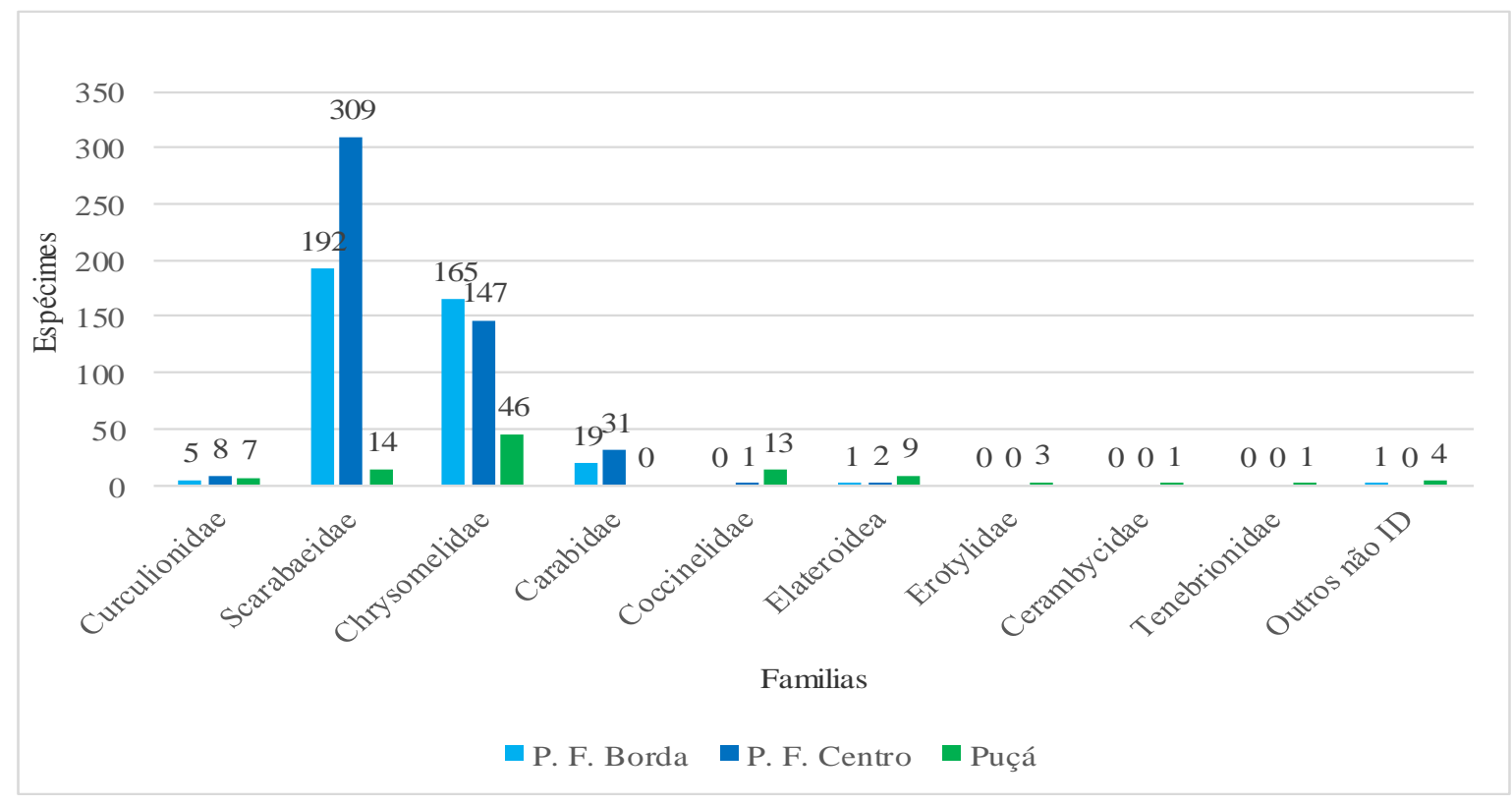

Fonte: Pinheiro Júnior (2021).

Com relação ao quantitativo de centro e borda, as famílias Scarabaeidae e Chrysomelidae foram as mais representativas nos dois ambientes, no entanto, a diferença entre borda e centro da família Scarabaeidae é significativamente maior do que a da família Chrysomelidae. De forma geral, estes resultados se assemelham ao encontrado no trabalho de Magalhães et al. (2015), onde utilizaram armadilhas do tipo pitfall e guarda-chuva entomológico, encontrando as famílias Scarabaeidae, Curculionidae e Carabidae como as mais abundantes.

Considerando o tipo de armadilha, Moura (2007) em coletas também utilizando armadilhas de queda, encontrou grande quantidade de uma morfoespécie de Carabidae, resultado este, semelhante ao encontrado no presente estudo, onde os carabideos apresentaram-se como a terceira família mais representativa e não foram representados na coleta por busca ativa.

Já com relação a diferença de centro e borda, a pesquisa de Cajaiba \& Silva (2015) com coleópteros do 
solo, aponta que a representatividade destes na borda é muito superior à da área de centro, porém, com uma menor diversidade e maior dominância, tal resultado, difere bastante do encontrado neste trabalho, pois a nossa representatividade de coleópteros no centro é maior do que a de borda.

Os índices de diversidade dos espécimes capturados na borda (Tabela 1), no centro (Tabela 2) e coletados por busca ativa (Tabela 3) foram calculados com o objetivo de conhecer a diversidade destes besouros nos diferentes locais, constatando que as armadilhas pitfall foram mais eficientes na captura dos espécimes, no entanto, os locais em que as armadilhas foram dispostas apresentam variação de diversidade e abundância, sendo necessário calcular o teste t-Student entre esses dois locais.

Tabela 1 - Valores referentes aos índices de diversidade de família capturados com armadilhas do tipo pitfall em borda. BORDA

\begin{tabular}{lr}
\hline Número de famílias (S) & 5 \\
Número total de indivíduos & 382 \\
Índice de Simpson $(\lambda)$ & 0.5582 \\
Índice de Shannon-Wiener (H') & 0.9299 \\
\hline \multicolumn{2}{c}{ Fonte: Pinheiro Júnior (2021). }
\end{tabular}

Tabela 2 - Valores referentes aos índices de diversidade de família capturados com armadilhas do tipo pitfall no centro. CENTRO

\begin{tabular}{lr}
\hline Número de famílias $(S)$ & 6 \\
Número total de indivíduos & 498 \\
Índice de Simpson $(\lambda)$ & 0.5237 \\
Índice de Shannon-Wiener (H') & 0.9301
\end{tabular}

Fonte: Pinheiro Júnior (2021).

Tabela 3 - Valores referentes aos índices de diversidade de família capturados com busca ativa. PUÇÁ

\begin{tabular}{lr}
\hline Número de famílias (S) & 8 \\
Número total de indivíduos & 94 \\
Índice de Simpson $(\lambda)$ & 0.7033 \\
Índice de Shannon-Wiener (H') & 1.532 \\
\hline \multicolumn{2}{c}{ Fonte: Pinheiro Júnior (2021). }
\end{tabular}

Os valores mostram que a riqueza de famílias (S) e os índices de diversidade de Simpson ( $\lambda$ ) e Shannon ( $\left.H^{\prime}\right)$ foram baixos para a borda, evidenciando a especificidade dessa armadilha em capturar besouros de solo ou ainda remetendo as características do efeito de borda. Do mesmo modo, estes mesmos índices foram baixos 
para a região do centro, porém, o número de espécimes capturados foi relativamente maior.

Com a realização do teste $\mathrm{t}$-Student calculado entre as armadilhas de borda e centro, encontramos $\mathrm{p}=$ 0,38 com resultado, demostrando que não há uma diferença significativa entre os índices de diversidade nos ambientes considerados. Estudos como o de Magalhães et al. (2015) também mostrou baixa diversidade nos dois locais pesquisados, não apresentando diferenças significativas entre suas diversidades, mesmo apresentando diferenças na quantidade de indivíduos. Isso demonstra, que a abundância de indivíduos não afeta diretamente a diversidade de espécimes.

Desse modo, podemos constatar que mesmo o número de espécies e famílias sofrendo variações de acordo com o nível de antropização do local, a diversidade pode variar pouco, pois está diretamente ligada ao tamanho da amostra, visto que, ambientes mais diversificados como a área de floresta, podem se apresentar com maior disponibilidade de recursos alimentares e tendem a permitir a coexistência e estabelecimento de diferentes espécies da fauna entomológica (Martins, Martins \& Souza, 2020).

\section{CONSIDERAÇÕES FINAIS}

O estudo revela que embora haja diferença no quantitativo de espécimes coletados nos diferentes ambientes (borda, centro e coleta ativa), os índices de diversidade entre estes locais não expressam valores com diferenças significativas, assim como, não houve grandes diferenças entre os períodos (meses) de coleta, apresentado estabilidade nos valores encontrados. No entanto, os números encontrados para a borda, por serem inferiores aos de centro, podem indicar a presença do efeito de borda, sendo necessário com isso, a realização de novos estudos, considerando maior esforço amostral e monitoramento da área para que seja realizado o monitoramento da diversidade destes indivíduos no local.

\section{AGRADECIMENTOS}

Agradecemos ao Laboratório de Biodiversidade e Conservação (LABICON) do Instituto Federal do Pará Campus Abaetetuba.

\section{REFERÊNCIAS}

Amado, S. F. A. (2012). Ocorrência e parâmetros ecológicos de coleópteros degradadores da madeira em fragmento de mata secundária e plantio de Pinus sp. no Campus de Seropédica da UFRRJ. Universidade Federal Rural do Rio de Janeiro. Instituto de Florestas. Seropédia, Rio de Janeiro. https://repositorio.ufsc.br/bitstream/handle/123456789/189143/TCC Eng Florestal AL 2018.pdf?sequence $\equiv 3 \&$ is Allowed $=\mathrm{y}$.

Berenguer, E., Ferreira, J., Gardner, T. A., Aragão, L. E. O. C., Camargo, P. B., Cerri, C. E., Durigan, M., Oliveira Junior, R. C., Vieira, I. C. G., Barlow, J. (2014). A large-scale field assessment of carbon stocks in human-modified tropical forests. Global change biology, 20(12), 3713-3726. 
Brasil, M. M. A. (2016). Quinto relatório nacional para a convenção sobre diversidade biológica: Brasil /Ministério do Meio Ambiente: Secretária de Biodiversidade e Florestas. Brasília, (240). https://www.terrabrasilis.org.br/ecotecadigital/images/abook/pdf/2017/Marco/Mar.17.39.pdf.

Burivalova, Z., Lee, T. M., Giam, X., Sekercioğlu, C. H., Wilcove, D. S., Koh, L. P. (2015). Avian responses to selective logging shaped by species traits and logging practices. proceedings of the royal society of london b: biological sciences, 282(1808).

Campos, R. C. (2012). Besouros Indicadores (Coleoptera, Scarabaeinae) na Avaliação de Alteração Ambiental em Fragmentos de Mata Atlântica Contíguos a Cultivos de Milho Convencional e Transgênico. 101 Dissertação (mestrado em ecologia) Universidade Federal de Santa Catarina, Florianópolis. https:/ / repositorio.ufsc.br/bitstream/handle/123456789/96204/302913.pdf? sequence=1\&isAllowed $=\mathrm{y}$.

Cajaiba, R. L., Silva, W. B. (2015). Abundância e Diversidade de Coleópteros de Solo em Fragmentos de Capoeira ao Entorno da Zona Urbana do Município de Uruará-PA, Brasil. EntomoBrasilis, 8(1), 30-37.

Casari, A.S., Ide, S. (2012). Insetos do Brasil: diversidade e taxonomia. Ribeirão Preto: Holos Editora.

Carvalho, R. S. (2011). Bioindicadores de qualidade edáfica com base na macrofauna para monitoramento e remediação de áreas degradadas e em transição agroecológica. in: Medeiros, C. A. B.; Carvalho, F. L. C.; Strassburger, A. S. (ed.). Transição Agroecológica - Construção Participativa do Conhecimento Para a Sustentabilidade: Projeto Macroprograma 1: Resultados de Atividades 2009-2010. Pelotas: 165-169.

Coelho, R. M. P. (2000). Fundamentos em ecologia. Porto Alegre: Artmed.

Costa, C. (2000). Estado de Conocimiento de los Coleoptera Neotropicales. Zaragoza: Sociedad Entomológica Aragonesa.

Costa, C., Vanin, S. A., Casari-Chen, S. A. (1988). Larvas de Coleoptera do Brasil. São Paulo: Museu de Zoologia.

Couto, R. C. S. (2020). Saúde e Ambiente na Amazônia Brasileira. Novos Cadernos NAEA. 23(3), 167-178.

Davis, A. J., Holloway, J. D., Huijbregts, H., Krikken, J., Kirk-spriggs, A. H., Sutton, S. L. (2001). Dung beetles as indicators of change in the forests of northern borneo. Journal of applied ecology, 38(1), 593-616.

Fernandes, F., Alves, S. S., Santos, H. F., Rodrigues, W. C. (2011). Staphylinidae e Silphidae (Coleoptera) como Potenciais Famílias Bioindicadoras de Qualidade Ambiental. Revista Eletrônica TECCEN, 4(3), 17-32.

França, L. C., Marini, M. Â. (2009). Teste do efeito de borda na predação de ninhos naturais e artificiais no Cerrado. Sociedade Brasileira de Zoologia, 26(2), 241-250.

Garlet, J., Costa, E. C., Boscardin, J. (2016). Levantamento da Entomofauna em Plantios de Eucalyptus ssp. Por meio de Armadilha luminosa em São Francisco de Assis - RS. Revista Ciências Florestal, 26(2).

Godinho Junior, C. L. (2011). Principais Famílias, Aspectos Biológicos e Morfológicos. In: Besouros e Seu Mundo. Rio de Janeiro: Technical Books, 155-172.

Gondim, M. J. C., Santos, L. A. S. (2013). Ações para organização de uma coleção didática de zoologia em uma escola de Uberlândia, MG. Revista Ciência em Extensão, São Paulo, 9(2), 19-27.

Gullan, P. J., Cranston, P. S. (2017). Insetos: fundamentos da entomologia. 5. Ed. Rio de Janeiro: Roca, 912. 
Hammer, O., Harper, D.A.T. \& Ryan, P. D. (2013). PAST: Paleontological Statistics software package for education and data analysis. http://https://folk.uio.no/ ohammer/past.

Hora, N., Fonseca, M. J. C. F., Sodré, M. N. R. (2015). Biodiversidade e Conservação; um olhar sobre a forma dos licenciandos de biologia. Revbea, São Paulo, 10(1), 56-74.

Lima, L. F. (2012). Efeito de Borda Sobre a Assembleia de Plantas Herbáceas em um Fragmento de Florestas Atlantica, Alagoas, Brasil. Dissertação Mestrado (Mestrado em Botânica) -Pró Reitoria de Pós-Graduação Universidade Federal Rural de Pernambuco, 93. http://www.bibliotecaflorestal.ufv.br/bitstream/handle/123456789/8981/Dissertacao Liliane $\% 20$ Ferreira $\% 20$ Lima.pdf?sequence $=1$.

López, A. B., Alkemade, R., Schipper, A. M., Ingram, D. J., Ver-Weij, P. A., Eikelboom, J. A. J. (2017). Huijbregts, M.A.J. The impact of hunting on tropical mammal and bird populations. Science. 356(6334), 180-183.

Machado, C. J. S., Vilani, R. M., Franco, M. G., Lemos, S. D. C. (2013). Legislação ambiental e degradação ambiental do solo pela atividade petrolífera no Brasil. Desenvolvimento e Meio Ambiente, Curitiba, 28(1), 41-55.

Magalhães, C. R. I., Oliveira, C. R. F., Oliveira, C. H. C. M., Nascimento, A. R. L. (2015). Biodiversidade de coleópteros predadores em áreas de Caatinga (Fazenda Saco, Serra Talhada - PE). Enciclopédia Biosfera, 11(21), 2068-2079.

Martins, L. M. O, Martins, W. M. O, Souza, E. A. (2020). Diversidade de insetos edáficos detritívoros na área experimental do IFAC-Campus Cruzeiro do Sul. Scientia Naturalis, Rio Branco, 2(1), 119-132.

Marinoni, R. C. (2001). Os grupos tróficos em Coleóptera. Revista Brasileira de Zoologia. Curitiba, 18(1), 205-224.

Metzger, J. P. (2012). Estrutura da paisagem: o uso adequado de métricas. In: Cullen Júnior, L.; Rudran, R.; Valladares-padua, C.P.C. Métodos de estudos em Biologia da Conservação e Manejo da Vida Silvestre. Curitiba: Universidade Federal do Paraná. 19-652.

Moreira, A. F. C. (2015). Classificação e identificação: classe insecta. In:_. Insetos: Manual de coleta e identificação. Rio de Janeiro: Technical Books, 126 - 136.

Moura, N. G., Lees, A. C., Andretti, C. B., Davis, B. J. W., Solar, R. R. C., Aleixo, A., Barlow, J., Ferreira, J., Gardner, T. A. (2013). Avian biodiversity in multiple-use landscapes of the Brazilian Amazon. Biological Conservation, 167(1), 339-348.

MOURA, L. A. Coleópteros terrestres. (2007). In: BECKER, F. G.; RAMOS, R. A \& MOURA, L. A. (eds.). Biodiversidade. Regiões da Lagoa do Casamento e dos Butiazais de Tapetes. Brasilia: Ministério do Meio Ambiente e Fundação Zoobotânica do Rio Grande do Sul, 2007. 387 p.

Nascimento, M. I., Poggiani, F., Durigan, G., Iemma, A. F., Silva Filho, D. F. (2010). Eficácia de barreira de eucaliptos na contenção do efeito de borda em fragmento de floresta subtropical no estado de São Paulo, Brasil. Scientia Forestalis, Piracicaba, 38(86), 191-203.

Oliveira, M. A., Gomes, C. F. F., Pires, E. M., Marinho, C. G. S., Lucia, T. M. C. D. (2014). Bioindicadores ambientais: insetos como um instrumento desta avaliação. Revista Ceres, Viçosa, 61(1), 800-807.

Otavo, S. E., Parrado, Á. R., Noriega, J. A. (2013). Superfamília Scarabaeoidea (Insecta: Coleoptera) como elemento bioindicador de perturbación antropogénica em um Parque Nacional Amazónico. Revista Biologia Tropical, 61(2), 735-752. 
Pedroso Júnior, N. N., Murrieta, R. S. S., Adams, C. (2008). A agricultura de corte e queima: um sistema em transformação. Ciências Humanas, Belém, 3(2), 153-174.

Pimenta, M. (2011). Chrysomelidae (Insecta/Coleoptera) como bioindicadores de Qualidade Ambiental em Áreas de Cerrado no Estado de Goiás - Brasil. Tese (Doutorado Ciências Ambientais) - Universidade Federal de Goiás, Goiânia, 144. https://repositorio.bc.ufg.br/tede/bitstream/tde/337/1/Tese\%20Mayra\%20Pimenta.pdf.

Quinteiro, T., Lopes, J., Martins, I. C. F. (2012). Diversidade de Carabidae (Coleoptera) amostrados em áreas de reflorestamento de mata ciliar e fragmento florestal, no Estado do Paraná. Entomo Brasilis, 5(3), 217 - 222.

Rafael, J. A., Melo, G. A. R., Carvalho, C. J. B., Casari, S. A., Constantino, R. (2012). Insetos do Brasil: diversidade e taxonomia. Ribeirão Preto, Holos Editora, 2012, p. 813.

Rocha, C. S. (2010). Ocorrência de coleópteros degradadores de madeira sob influência de diferentes espaçamentos, em plantios florestais. Seropédica. Trabalho de conclusão do curso - Universidade Federal Rural do Rio de Janeiro, p. 39.

Rodríguez, V. A., Rojas, C., Vázquez, R. A. (2016). Saldaña; STONER, Kathryn E. Landscape composition is more important than landscape configuration for phyllostomid bat assemblages in a fragmented biodiversity hotspot. Biological Conservation, 198(1), 84-92.

Saldanha, M. A., Costa, E. C., Machado, L. M., Machado, D. N., Silva, J. M., Pedron, L.,

Galvan, C. F., Silva, B. C. (2020). Distribuição vertical e diversidade de coleópteros em povoamento de Pinus taeda L. (Pinaceae). Caderno de Ciências Agrárias, 12(s.n), 1-11.

Santos, L. R., Benchimol, M., Mayfield, M. M., Faria, D., Pessoa, M. S., Talora, D. C., Mariano Neto, E., Cazetta, E. (2017). Functional decay in tree community within tropical fragmented landscapes: Effects of landscape-scale forest cover. PLOS ONE, 12(4), 1-18.

Souza Júnior, C. M., Siqueira, J. V., Sales, M. H., Fonseca, A. V., Ribeiro, J. G., Numata, I., Cochrane, M. A., Barber, C. P., Roberts, D. A., Barlow, J. (2013). Ten-year Landsat classification of deforestation and forest degradation in the Brazilian Amazon. Remote Sensing, 5(11), 5493-5513.

Spiller, M. S., Spiller, C., Garlet, J. (2018). Arthropod bioindicators of environmental quality. Revista Agroambiente On-line, Boa Vista, 12(1), 41-57.

Silva, P. G., Silva, F. C. G. (2011). Besouros (Insecta: Coleoptera) utilizados como bioindicadores. Revista Congrega Urcamp, 5(s.n), 1-16.

Teixeira, C. C. L., Hoffmann, M., Silva Filho, G. (2009). Comunidade de Coleoptera de solo em remanescente de Mata Atlântica no estado do Rio de Janeiro, Brasil. Biota Neotropica, 9(4).

Triplehorn, C. A., Johnson, N. F. (2011). Estudo dos insetos. $7^{\circ}$ Ed. São Paulo: Cengage Learning, p. 809.

Viviani, V. R., Rocha, M. Y., Hagen, O. (2010). Fauna de Besouros Bioluminescentes (Coleoptera: Elateroidea: Lampyridae; Phengodidae, Elateridae) Nos Municípios de Campinas, Sorocaba - Votorantim e Rio Claro Limeira (SP, Brasil): Biodiversidade e Influência da Urbanização. Biota Neotropical, 10(2), 103-116.

Wink, C. Guedes, J. V. C., Fagundes, C. K., Rovedder, A. P. (2005). Insetos edáficos como indicadores da qualidade ambiental. Revista de Ciências Agroveterinárias, Lages, 4(1), 60-71. 